Intel Collaborative Research Institute - Sustainable Connected Cities Peer-reviewed author version

SCHOENING, Johannes; Rogers, Yvonne; Bird, Jon; Capra, Lucia; McCann, Julie A.; Prendergast, David \& Sheridan, Charles (2012) Intel Collaborative Research Institute - Sustainable Connected Cities. In: Paternò, Fabio; De Ruyter, Boris; Markopoulos, Panos; Santoro, Carmen; van Loenen, Evert; Luyten, Kris (Ed.). Ambient Intelligence: Third International Joint Conference, AMI 2012, Pisa, Italy, 13-15 November 2012, Proceedings, p. 364-372.

DOI: $10.1007 / 978-3-642-34898-3 \_28$

Handle: http://hdl.handle.net/1942/14321 


\title{
Intel Collaborative Research Institute - Sustainable Connected Cities
}

\author{
Johannes Schöning ${ }^{1,2,4}$, Yvonne Rogers ${ }^{1,2}$, Jon Bird $^{1}$, Licia Capra $^{1,2}$, Julie A. \\ McCann $^{2,3}$, David Prendergast ${ }^{2}$, and Charles Sheridan ${ }^{2}$ \\ 1 University College London, Gower Street, London, WC1E 6BT, UK \\ ${ }^{2}$ Intel Collaborative Research Institute - Sustainable Connected Cities, London, UK \\ ${ }^{3}$ Imperial College London,Huxley Bldg, London, SW7 2AZ, UK \\ 4 Hasselt University, Wetenschapspark 2, 3590 Diepenbeek, Belgium
}

\begin{abstract}
Cities are places where people, meet, exchange, work, live and interact. They bring people with different interests, experiences and knowledge close together. They are the centres of culture, economic development and social change. They offer many opportunities to innovate with technologies, from the infrastructures that underlie the sewers to computing in the cloud. One of the overarching goals of Intel's Collaborative Research Institute on Sustainable Connected Cities is to integrate the technological, economic and social needs of cities in ways that are sustainable and human-centred. Our objective is to inform, develop and evaluate services that enhance the quality of living in the city.
\end{abstract}

\section{Motivation}

There are many visions of the future focusing on smart cities, future cities and ambient intelligence. An underlying theme is that cities become increasingly embedded with invisible (and some still visible) technologies, sensitive and responsive to peoples needs that deliver advanced functions, services and experiences [1], following the vision of Mark Weise [2]. Intel's Collaborative Research Institute on Sustainable Connected Cities ${ }^{5}$ is concerned with enhancing and changing how people live, interact and engage with cities. Our goal is to enhance city sustainability and improve citizen well being.

Our perspective in the Sustainable Connected Cities Institute is to be humancentred. We have wide-ranging expertise and background in user experience, interaction design, ethnography, together with research in the built environment, commerce, engineering, anthropology, the arts, and social psychology. We also work as inter-disciplinary teams that can make a real change to enrich and extend city dwellers lives. This fits in with Bells vision for 2012 [3] of computers not just acting on our behalf and anticipating what we want but also enabling people to be more creative, using state of the art computer technologies and toolkits.

\footnotetext{
${ }^{5}$ http://www.connected-cities.org
} 


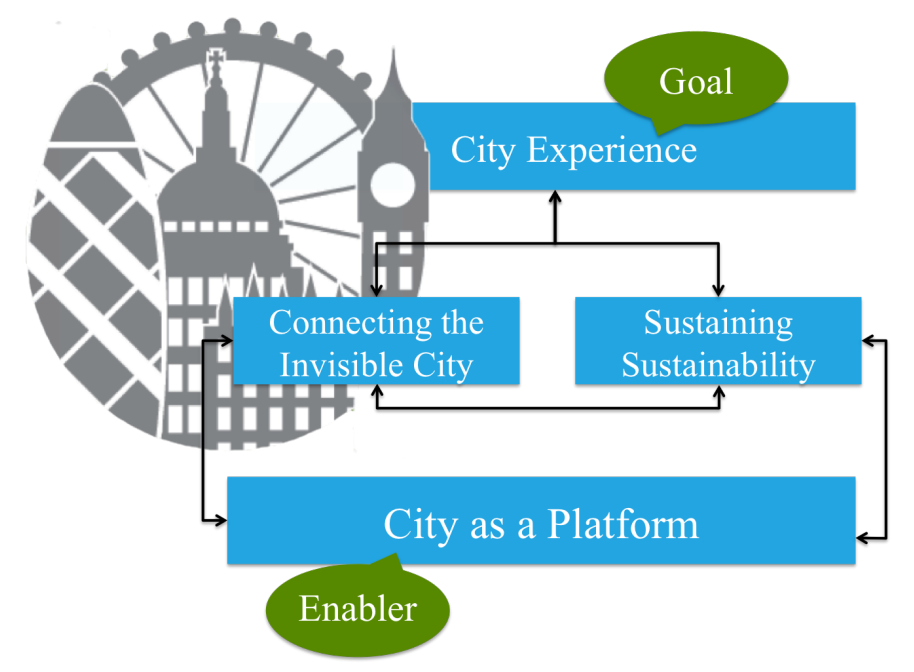

Fig. 1. Intel's Collaborative Research Institute on Sustainable Connected Cities thematic research agenda

There are major challenges that future cities will face not least a huge increase in the populations living in urban areas. According to a United Nation report [4] every second the global urban population grows by 2 people. Therefore the urban population is expected to increase from 3.6 billion people in 2011 to 6.3 billion in 2050. In 2020 more than 700 cities will exist with populations of +1 million; today we have just 500 cities with populations of +1 million. The exploding urban population growth creates unprecedented challenges, among which provision for water and sanitation are the most pressing and painfully felt when lacking [5]. Cities cannot be sustainable without ensuring reliable access to safe drinking water and adequate sanitation.

A focus will be on the techniques and solutions to tackle these future challenges. We will develop and exploit pervasive and sensing technologies, analytics and new interfaces, putting humans at the centre of technological developments. Our approach is to address four main themes (see figure 1)

- City Experience: How do we enhance the City Experience and communicate services?

- City as a Platform: How do we create the digital platform of the city from sensor/edge to cloud?

- Sustaining Sustainability: How to sustain behavioural change?

- Connecting the Invisible City: How do we visualize the Human-Environment Interface? 


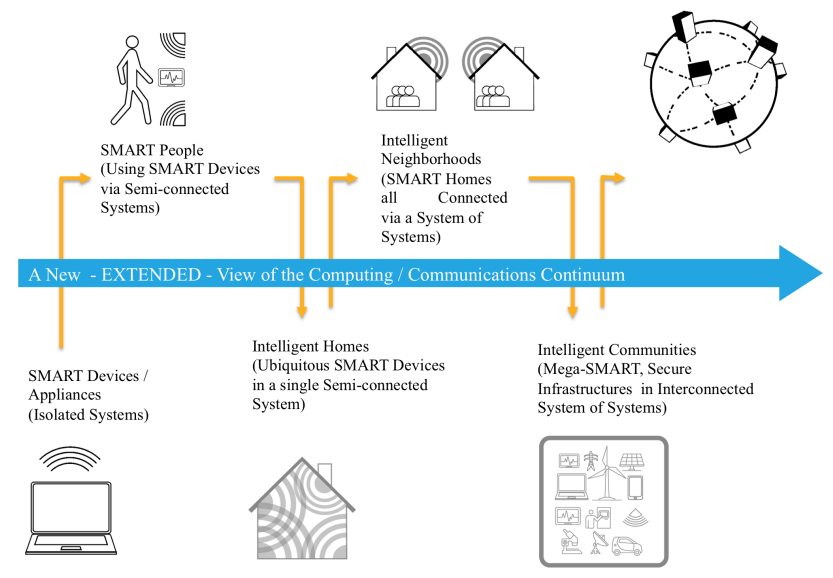

Fig. 2. Path to Connected Cities

\section{Connected Cities}

We consider how cities will become instrumented environments that serve the needs of their citizens (see figure 2). Inspired by the Internet of Things (IoT), a number of projects are beginning to develop connected products that can be embedded in the environment and people's homes. In addition, our working, learning and recreation places are becoming more equipped with technologies and sensors. Secure infrastructures are essential for such interconnected systems of systems. Besides the question of how to support such an elastic city platform from sensor/edge to cloud (see [6]), it is necessary to research how citizens interface with such an interconnected system of technologies and services.

Another growing concern, given limited resources, is how cities can be more sustainable and encourage their citizens to use utilities (water, electricity, gas, etc.) in more resourceful ways.

\section{Sustaining Sustainability}

Helping citizens adapt their behaviour in order make cities more sustainable involves increasing their awareness of how they live and then encourage changing habits, at an individual, family, local community and city level. This requires adopting a broad unit (ranging from the individual to communities at large) of analysis: considering the needs of the individual city dweller, families, whole neighbourhoods, councils, and communities at large.

There are a number of behavioural change techniques that have been identified in social psychology and behavioural science. One of the most well known 
approaches used in behavioural therapy is the Transtheoretical Model. This predicts a persons success or failure in achieving a proposed behaviour (e.g., dieting) in terms of their preparedness to change as described by Prochaska [7]. Despite its popularity, however, recent reviews of a large number of empirical studies have shown little evidence for the effectiveness of stage-based interventions as a basis for behavioural change (e.g., Aveyard [8]).

Other promising techniques that have been suggested for use in behavioural change include peer pressure, positive reinforcement, and social norms [9]. Peer pressure has been used to help people change their behaviour for a variety of health-related areas, such as alcoholism, obesity and smoking. Computer applications that have tried to capitalise on this approach have displayed data, such as the amount of exercise completed on a mobile device, comparing how members of a group are doing relative to each other (e.g [10]). An innovative prototype, that used both positive reinforcement and peer pressure, was the WaterBot system, designed to help householders reduce their usage of water in their homes [11]. There is much evidence to suggest that people are wasteful with water, often leaving the tap running continuously for long periods of time while cleaning their teeth or washing. The research team thought that the use of monitoring technology could help persuade householders to change their behaviour and be more conservative in their water usage. To this end, they used the theory of positive reinforcement to inform their design, which states that activities are likely to be repeated if some kind of reward is given occasionally and randomly (similar to the reward system used in slot machines). A sensor-based system was developed where positive auditory messages and chimes were sounded when the tap was turned off. Here, the idea was to encourage peer pressure and for the members of the household to talk to each other about their water usage.

Since this study, the global concern about climate change has led a number of researchers to design and evaluate various energy sensing devices that display real-time feedback. A goal is to find ways of helping people reduce their energy consumption (and is part of a larger research agenda called sustainable HCI, e.g., Mankoff [12]). A focus is on persuading people to change their everyday habits with respect to environmental concerns, such as reducing their own carbon footprint. The effect of social norms on people's energy consumption has also begun to be studied. For example, Schultz et al. [13] looked at how people behaved when provided with information that compared their electricity usage to the neighbourhood average. They found that providing the average had a big impact on households own energy consumption. As hoped, households above the average tended to decrease their consumption but those using less electricity than average tended to increase their consumption. The study suggested that such boomerang effects could be counteracted by providing households with further salient information that was emotive. When provided with an emoticon along with the numerical information about their energy usage, households using greater than average decreased their consumption more if they were given a sad icon and, significantly, households using less energy than average continued to do so if they received a smiley icon. 
In the last few years, there have been many research and council led initiatives in cities across the world to reduce energy consumption, from reducing street lighting to implementing smart metering. Some have shown impressive results. However, many of these have proven to be short-lived. Moreover, there is a tendency to return to old habits once the local champion, publicity intervention, etc. have been taken away. A key question, which we will address at the centre, is: How can sustainable behaviour in its various forms be sustained over a long period of time, preferably indefinitely? What mix of policies and technologies can be used to best effect? Which behaviours are most amenable? How do communities take on the sustainable challenge themselves and understand what it takes?

We intend to develop a science of behavioural change that is predictive and generalizable to different contexts; longitudinal empirical studies will be carried out to investigate long-term effects. We further argue that the efficacy of the techniques and methods used will be affected by how ethical they are. The aim of this theme is to investigate how behaviour can be changed effectively, is socially acceptable and will persist over a variety of contexts and settings. The overarching goal is to engage citizens proactively with new kinds of technologically augmented information in different aspect of their lives and cities. Moreover, we intend to involve them directly in identifying problem behaviours they care about in city life, generating prototype designs and actively participating in the evaluation studies.

The research intends to push the frontiers of the science of behavioural change by systematically addressing many of the assumptions and unknowns in this exciting new field, using a three-pronged approach:

- designing and implementing a range of new pervasive technologies that can facilitate behaviour change by operationalizing theories from behavioural economics and social psychology

- assessing how new kinds of information and multimodal real-time feedback are best delivered by pervasive technologies and which are the most effective techniques for different contexts and behaviours

- ascertaining whether and how salient information can lead people to change their behaviour in both the short-term and the long-term.

A key objective is to show how different combinations of technologies, behaviour techniques and salient information can systematically facilitate behaviour change, with a focus on those behaviours that either have not been considered before or have been resistant to change using other methods. A further goal is to design technologies that are affordable and customisable so that they can be adopted by individuals and communities who have a problem they wish to address - for example, they may wish to reduce vandalism in their neighbourhood, encourage more volunteering or increase local shopping. 


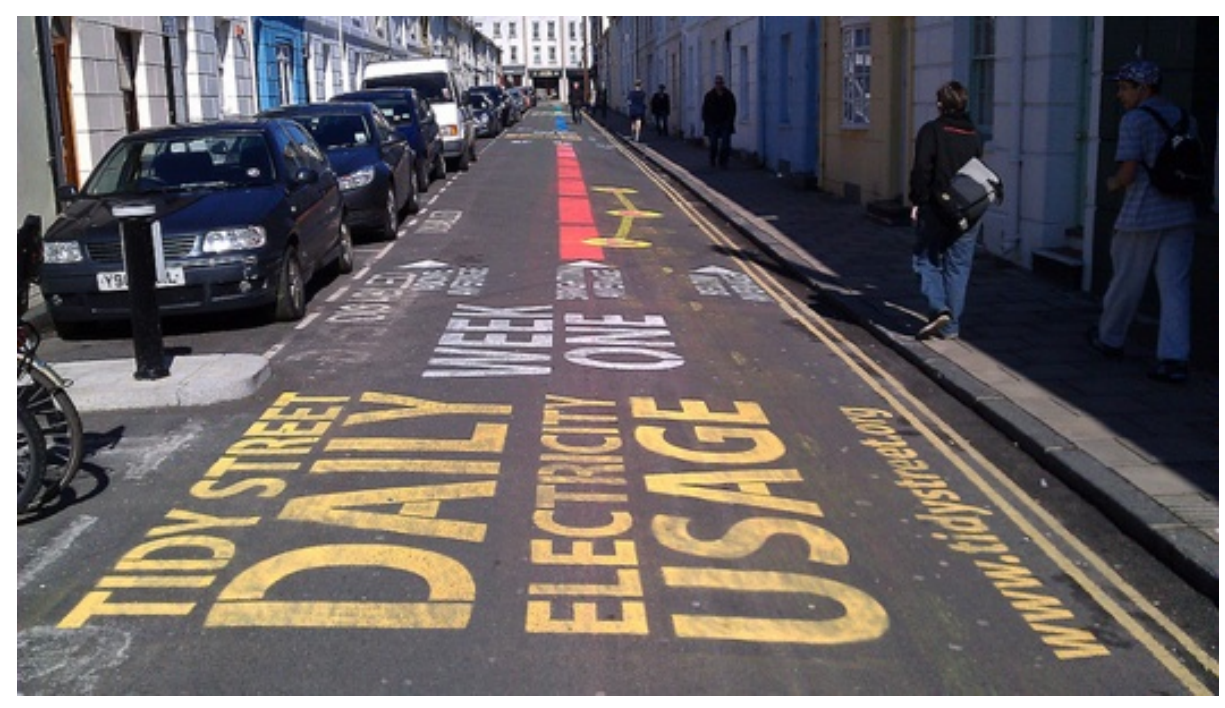

Fig. 3. Tidy Street: Measuring and Publicly Displaying Domestic Electricity Consumption (Photo taken by Nora O'Murchu)

\subsection{Tidy Street: An example of Sustaining Sustainability}

Our own research has shown that publicly displaying households electricity consumption can have a significant impact on their energy usage [14]. Participants reported an increased awareness of their electricity usage together with reduced their electricity usage by $15 \%$. A display sprayed with chalk on the street based on social norms, that represent both the communitys average compared to the city at large, can be seen in figure 3 .

\section{Connecting the Invisible City: Visualizing the Human-Environment Interface}

The Connecting the Invisible City theme focuses on how technology can help recognize, leverage, and support the out-of-sight, hidden or forgotten resources of urban environments from volunteers to subterranean water systems and other underlying city infrastructures. In future cities a lot of data streams and information will be embedded and stored within their infrastructure. Besides determining new ways of how to store, save and update all this information within complex infrastructures, new ways of thinking about and analysing information will need to be developed. A fundamental question is what novel multimodal interfaces and interactions are required to encourage participation of citizens, business and government. Over the last few years, advances in graphical interfaces (e.g., the iPhone UI), speech recognition (e.g. Siri), gesture and handwriting recognition 
(e.g., Kinect), together with the arrival of the mobile broadband, smartphones, sensor technologies, and an assortment of other new technologies providing large and small interactive displays, have changed the face of human-computer interaction [15].

A challenge is to develop displays, services and apps that can visualize the invisible information flows in future cities and help people to make informed decisions during their daily routines. We will investigate and develop a framework for Human Environment Interfaces (HEI) that lets individuals and groups engage with the information available in the city. But how do we visualize the HEI? What resources are visible/invisible? What actors are invisible/invisible? How and where should city information be represented? Possible visualisations include aggregation of quantified self and community data, via ambient displays, mobile devices and public signage. Our research will focus on the following topics:

- The development of novel interaction techniques which will afford interaction, to help participants to discover services and data around them.

- The development of services or interfaces that turn data into information and help people to make better informed decisions.

- The development of technologies to encourage sustainable behaviour through ambient and invisible interfaces which capture information relating to citizens behaviour.

- The development of interaction techniques that connect people to their cities..

The topics are wide-ranging from making invisible data visible, turning data into useful information, supporting sustainable behaviour and helping citizens to experience their cities in new ways. We will focus on providing information and experiences instead of pure data and facts. The recent range of technological developments described above has encouraged different ways of thinking about interaction design. Researchers and developers have combined the physical and digital in novel ways, resulting in mixed realities, augmented realities, tangible interfaces, and wearable computing. Overall, it is important to design multi-modal techniques (addressing the visual, hearing, and haptic senses), which provide the right degree of abstraction for each citizen in each context.

\subsection{PhotoMap: An example for new ways of interaction within cities}

In the following we try to describe, based on our own research, new ways of interacting with a city platform. Other examples (looking at different perspectives on this problem) are described in $[16,17]$. In many mid-to large-sized cities public maps are ubiquitous. These public maps help to facilitate orientation and provide special information to tourists but also to locals who just want to look up an unfamiliar place while on the go. These maps offer many advantages compared to mobile maps from services like Google Maps Mobile or Nokia Maps. They often show local landmarks and sights that are not shown on standard 
digital maps. Often these YOU ARE HERE (YAH) maps are adapted to a special use case, e.g. a zoo map or a hiking map of a certain area and tailored to a specific usage context. Being designed for a fashioned purpose these maps are often aesthetically well designed and their usage is therefore more pleasant.

But how do we bring this information to the city platform to encourage sustainable behaviour? The Photomap application [18] is a novel technique and application that uses images of YAH maps taken with a GPS-enhanced smart phone as background maps for on-the-y navigation tasks. We have shown that pedestrians can georeference the taken images with sufcient accuracy to support navigation tasks and feed data to the platform.

\section{Summary}

The aim of the new institute is to create and realize a compelling vision of a sustainable future made possible by adaptive technologies that optimize resource efficiency, enable new services and support the quality of life of urban inhabitants. Our vision is to enhance city sustainability and improve citizen well-being. For our research the city of London will be the main testbed. It will be important to research what technologies are needed that can best identify, model, and promote understanding of the impacts of urban heat and climate change on the people and infrastructure. The project will provide new understandings and insights into how cities can be better instrumented and citizens better informed about the resources and utilities they use. Of course, London will be not the only testbed in Europe. Various cities exists that will face important challenges within the next few years. In addition the Urbanization will heavily effect other parts of the world, e.g. Africa and Asia, and will of course need attention on a global scale. We hope to collaborate with others to innovate in and with communities, within Europe and within other parts of the world, using sustainable affordable technologies that can transform cities.

\section{References}

1. Aarts, E.: Ambient intelligence: A multimedia perspective. Multimedia, IEEE 11(1) (2004) 12-19

2. Weiser, M.: The computer for the 21st century. Scientific American 265(3) (1991) 94-104

3. Bell, G., Dourish, P.: Yesterdays tomorrows: notes on ubiquitous computings dominant vision. Personal and Ubiquitous Computing 11(2) (2007) 133-143

4. Department of Economic: The Millennium Development Goals Report 2010. United Nations Publications (2010)

5. United Nations Development Program. Human Development Report Office: Human development report: Background papers. Human Development Report Office, United Nations Development Programme (2001)

6. McCann, J.: Engineering beauty with the beasties on wireless sensor networks. Perada Magazine (2008) 
7. Prochaska, J., Norcross, J.: Systems of psychotherapy: A transtheoretical analysis. Brooks/Cole Pub Co (2009)

8. Aveyard, P., Lawrence, T., Cheng, K., Griffin, C., Croghan, E., Johnson, C.: A randomized controlled trial of smoking cessation for pregnant women to test the effect of a transtheoretical model-based intervention on movement in stage and interaction with baseline stage. British journal of health psychology 11(2) (2006) 263-278

9. Consolvo, S., McDonald, D., Landay, J.: Theory-driven design strategies for technologies that support behavior change in everyday life. In: Proceedings of the 27th international conference on Human factors in computing systems, ACM (2009) 405-414

10. Toscos, T., Faber, A., An, S., Gandhi, M.: Chick clique: persuasive technology to motivate teenage girls to exercise. In: CHI'06 extended abstracts on Human factors in computing systems, ACM (2006) 1873-1878

11. Arroyo, E., Bonanni, L., Selker, T.: Waterbot: exploring feedback and persuasive techniques at the sink. In: Proceedings of the SIGCHI conference on Human factors in computing systems, ACM (2005) 631-639

12. Mankoff, J., Kravets, R., Blevis, E.: Some computer science issues in creating a sustainable world. Computer 41(8) (2008) 102-105

13. Schultz, P., Nolan, J., Cialdini, R., Goldstein, N., Griskevicius, V.: The constructive, destructive, and reconstructive power of social norms. Psychological Science 18(5) (2007) 429-434

14. Bird, J., Rogers, Y.: The pulse of tidy street: Measuring and publicly displaying domestic electricity consumption. In: Workshop on Energy Awareness and Conservation through Pervasive Applications (Pervasive 2010). (2010)

15. Preece, J., Rogers, Y., Sharp, H.: Interaction design: beyond human-computer interaction. Wiley (2002)

16. Böhmer, M., Hecht, B., Schöning, J., Krüger, A., Bauer, G.: Falling asleep with angry birds, facebook and kindle: a large scale study on mobile application usage. In: Proceedings of the 13th International Conference on Human Computer Interaction with Mobile Devices and Services, ACM (2011) 47-56

17. Schöning, J., Hecht, B., Starosielski, N.: Evaluating automatically generated location-based stories for tourists. In: CHI'08 extended abstracts on Human factors in computing systems, ACM (2008) 2937-2942

18. Schöning, J., Krüger, A., Cheverst, K., Rohs, M., Löchtefeld, M., Taher, F.: Photomap: using spontaneously taken images of public maps for pedestrian navigation tasks on mobile devices. In: Proceedings of the 11th International Conference on Human-Computer Interaction with Mobile Devices and Services, ACM (2009) 14 